Article

\title{
Study of Detector-Grade CdMnTe:In Crystals Obtained by a Multi-Step Post-Growth Annealing Method
}

\author{
Pengfei Yu ${ }^{1,2, *}$, Yongren Chen ${ }^{1}$, Wei Li ${ }^{1}$, Wenfei Liu ${ }^{1}$, Bin Liu ${ }^{1}$, Jie Yang ${ }^{1}, \mathrm{Kun} \mathrm{Ni}^{1}$, \\ Lijun Luan ${ }^{1}$, Jiahong Zheng ${ }^{1}$, Zhuo Li ${ }^{1}$, Min Bai ${ }^{1}$, Guodong Sun ${ }^{1}$, Hui Li ${ }^{1}$ and Wanqi Jie ${ }^{2}$ \\ 1 School of Materials Science and Engineering, Chang'an University, Xi'an 710061, China; \\ 2017131038@chd.edu.cn (Y.C.); 201531050204@chd.edu.cn (W.L.); 2016900950@chd.edu.cn (W.L.); \\ 201531050220@chd.edu.cn (B.L.); 201531050234@chd.edu.cn (J.Y.); 2016905225@chd.edu.cn (K.N.); \\ nmllj050@chd.edu.cn (L.L.); jhzheng@chd.edu.cn (J.Z.); lizhuo@chd.edu.cn (Z.L.); baimin@chd.edu.cn (M.B.); \\ sunguodong@chd.edu.cn (G.S.); huili@chd.edu.cn (H.L.) \\ 2 State Key Laboratory of Solidification Processing, Northwestern Polytechnical University, Xi'an 710072, \\ China; jwq@nwpu.edu.cn \\ * Correspondence: yupengfei@chd.edu.cn; Tel.: +86-29-8233-7340
}

Received: 8 August 2018; Accepted: 7 October 2018; Published: 11 October 2018

\begin{abstract}
A multi-step annealing method was successfully applied to inclusions reduction and resistivity improvement of CdMnTe:In (CMT:In) single crystals with high resistivity, including a Cd atmosphere annealing step followed by a Te atmosphere annealing step. After the $\mathrm{Cd}$ atmosphere annealing step, the density of Te inclusions was reduced distinctly, and it could be also decreased in the subsequent step of re-annealing under Te atmosphere. Both the resistivity and IR transmittance decreased notably after $\mathrm{Cd}$ atmosphere annealing, whereas they increased tremendously after re-annealing under a Te atmosphere. The reduction of full-width at-half-maximum (FWHM) and the increase of the intensity of the X-ray rocking curve indicated an improvement of the crystal quality. Meanwhile, after $\mathrm{Cd}$ atmosphere annealing, the increase of the intensity of the $\left(\mathrm{D}^{0}, \mathrm{X}\right)$ peak and the disappearance of the $\left(\mathrm{A}^{0}, \mathrm{X}\right)$ peak in photoluminescence $(\mathrm{PL})$ measurements suggested further that the crystal quality was improved. The detector performance was enhanced obviously after annealing. The higher the annealing temperature, the better the performance was. The detector fabricated by CMT:In slice (Cd atmosphere annealing at $1073 \mathrm{~K}$ for $240 \mathrm{~h}$ and Te atmosphere re-annealing at $773 \mathrm{~K}$ for $120 \mathrm{~h}$ ) with $9.43 \%$ energy resolution and $1.25 \times 10^{-3} \mathrm{~cm}^{2} / \mathrm{V} \mu \tau$ value had the best detector performance.
\end{abstract}

Keywords: CdMnTe:In; Te inclusions; resistivity; crystal quality; radiation detector

\section{Introduction}

The compound semiconductor material CdMnTe (CMT) has many applications for infrared (IR) detectors, solar cells, optical isolators and spintronic devices [1]. It is considered to be the substitute material of CdZnTe because of its optimal band-gap energy, fine mechanical stability, and near-unity segregation coefficient of Mn [2]. At present, CMT has attracted interest for the application of a room temperature radiation detector due to its wide band-gap, high resistivity, and good electron-transport properties [3-5]. However, CMT single crystals usually have some structure defects, especially Te inclusions, which will badly deteriorate the detector performance [6]. Therefore, in order to improve the crystal quality, researchers adopt post-growth annealing to reduce or eliminate these defects. However, few studies about the annealing of CMT crystals have been reported [7-10]. Generally, one 
annealing method only contains one step. For example, in order to reduce Te inclusions, CdTe-based crystals are generally annealed under a Cd atmosphere [11-13].

For CdTe-based room temperature radiation detectors, one very important property is resistivity. However, annealing in a $\mathrm{Cd}$ atmosphere will lead to an obvious decrease of the resistivity and the crystal will lose detection ability. How to increase the resistivity again is very important. As is well known, the Fermi-level of semiconductor material must be pinned at the mid band-gap position to obtain high resistivity. The deep-level centers in crystals are a key factor of obtaining high resistivity [14]. Moreover, the reduction of carrier concentration by Te annealing is another reason to obtain high resistivity. Yang et al. [15] used a two-step annealing method (Cd annealing followed by Te annealing) for CdZnTe. Te annealing results in abundant formation of $\mathrm{Cd}$ vacancies, which are acceptors, and then carrier concentration is decreased. In this paper, the aim is focused on the elimination of Te inclusions and keeping high-resistivity by optimal annealing conditions. The annealing of CMT:In crystals was adopted using a multi-step method, including a Cd atmosphere annealing step followed by a Te atmosphere annealing step. The effects of annealing temperature on the properties of CMT:In crystals were investigated. Moreover, the performance of CMT radiation detectors was also measured.

\section{Materials and Methods}

One $\mathrm{Cd}_{0.9} \mathrm{Mn}_{0.1} \mathrm{Te}$ :In (CMT:In) crystal ingot was grown using the modified vertical Bridgman method using high-purity raw materials of $\mathrm{Cd}(7 \mathrm{~N}), \mathrm{Mn}(5 \mathrm{~N})$, and $\mathrm{Te}(7 \mathrm{~N})$. The ingot was doped with $15 \mathrm{ppm}$ Indium (7N), equivalent to a concentration of $5 \times 10^{17}$ atoms $/ \mathrm{cm}^{-3}$. CMT wafers were cut from the ingot along (111) face of the ingot. The wafers were then diced into small slices with the dimensions of $10 \times 10 \times 2 \mathrm{~mm}^{3}$. The slices with a resistivity of about $10^{9} \Omega \cdot \mathrm{cm}$ were chosen for multi-step annealing treatment. Annealing was performed in a two-zone furnace. Before annealing, all the slices were polished with $\mathrm{MgO}$ suspension and then etched with $3 \% \mathrm{Br}_{2}-\mathrm{MeOH}$ solution. The multi-step annealing treatment was performed in two annealing steps, including a $\mathrm{Cd}$ atmosphere annealing step and a Te atmosphere annealing step. High-purity $\mathrm{Cd}(\mathrm{TN})$ and $\mathrm{Te}(7 \mathrm{~N})$ were used for the annealing sources. CMT could be considered as CdTe because of the low concentration of $\mathrm{Mn}$ in CMT. According to Li et al. [16], the annealing temperature must be chosen from $722 \mathrm{~K}$ to $1150 \mathrm{~K}$. Thus, annealing temperatures of $1073 \mathrm{~K}, 973 \mathrm{~K}$, and $873 \mathrm{~K}$ (higher than the melting point of Te $(723 \mathrm{~K})$ ) were adopted for $\mathrm{Cd}$ atmosphere annealing. The annealing time was chosen as $240 \mathrm{~h}$, according to our previous research [17]. The purpose of this step was to reduce or eliminate Te inclusions. The sources and the slices were fixed at different sides of quartz crucibles. The quartz crucibles were sealed under a vacuum of $10^{-5} \mathrm{~Pa}$ and then placed into a two-zone annealing furnace. When the annealing temperature on the side of the slice was $873 \mathrm{~K}$, the temperature on the side of the source was $813 \mathrm{~K}$. After annealing under a $\mathrm{Cd}$ atmosphere, the slices were re-annealed under a Te atmosphere. This step was aimed at enhancing the resistivity further. In order to reduce $\mathrm{Cd}$ evaporation in crystals, the annealing temperature of both slices and the source was chosen as $773 \mathrm{~K}$. The annealing experiment under a Te atmosphere was also carried out in a two-zone furnace and the annealing time was $120 \mathrm{~h}$.

The near-infrared spectrum (NIR) was tested by a Shimadzu UV-3150 UV-VIS-NIR spectrometer (Shimadzu, Tokyo, Japan) in the wavelength range of 1200 to $700 \mathrm{~nm}$ at room temperature. Te inclusions were observed by IR transmission microscopy (Micronviewer 7290A, Electrophysics Corporation, Fairfield, NJ, USA). IR transmittance was measured by a Nicolet Nexus 670 spectrometer (Thermo Electron Corporation, Waltham, MA, USA) in the wavenumber range from $4000 \mathrm{~cm}^{-1}$ to $500 \mathrm{~cm}^{-1}$. Photoluminescence (PL) measurement was carried out by a Triax 550 tri-grating monochrometer (HORIBA Jobin Yvon, Paris, France). An argon ion laser with a wavelength of $488 \mathrm{~nm}$ was used to excite the PL spectra. The measurement temperature was $10 \mathrm{~K}$. Meanwhile, the current-voltage (I-V) curves of samples were tested by Agilent 4155C (Agilent Technologies Inc., Santa Clara, CA, USA) at room temperature. The energy resolution of the detectors was measured with an ORTEC measurement system (AMETEK ORTEC, Oak Ridge, TN, USA) using a ${ }^{241} \mathrm{Am} \gamma$-ray source. 


\section{Results and Discussion}

\subsection{Effect of Multi-Step Annealing on the Concentration of $\mathrm{Mn}$}

NIR transmittance is a nondestructive and easy way for determining the relative content of Mn and the band gap of CMT. The typical NIR transmittance spectra of CMT:In crystals before and after annealing (Cd atmosphere $1024 \mathrm{~K} / 240 \mathrm{~h}$, Te atmosphere $773 \mathrm{~K} / 120 \mathrm{~h}$ ) are shown in Figure 1. It can be seen that the shapes of the transmittance spectra of CMT:In crystals before and after annealing are almost coincident except for the maximum transmittance. This indicates that the multi-step, post-growth annealing has almost no effect on the Mn element because Mn vapor pressure is low. Therefore, the band gap of $\mathrm{Cd}_{0.9} \mathrm{Mn}_{0.1} \mathrm{Te}$ :In does not change before and after annealing.

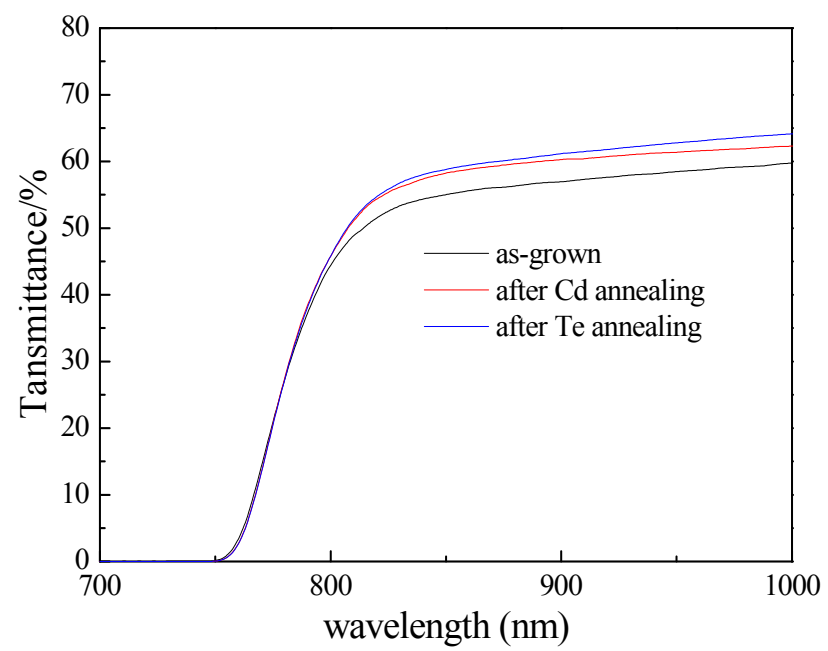

Figure 1. The typical near-infrared spectrum (NIR) transmittance spectra of CMT:In crystals before and after annealing (Cd atmosphere 1073K/240 h, Te atmosphere $773 \mathrm{~K} / 120 \mathrm{~h}$ ).

\subsection{Effect of Multi-Step Annealing on Te Inclusions}

Figure 2 shows the typical IR microscope image of Te inclusions in an as-grown CMT:In crystal. It can be observed that the density of Te inclusions is large. Te inclusions with the size about 1 15 $\mu \mathrm{m}$ are mainly near-triangular and hexagonal. Figure 3 shows IR microscope images of the CMT:In slices before and after annealing under a $\mathrm{Cd}$ atmosphere. The sample is observed in situ. For as-grown CMT:In slices (cut from the same wafer), the inclusion density is calculated to be $(2 \sim 3) \times 10^{5} \mathrm{~cm}^{-2}$ (Figure $3 \mathrm{~A}-\mathrm{C}$ ). After annealing at $873 \mathrm{~K}$ (Figure 3a), $973 \mathrm{~K}$ (Figure 3b), and $1073 \mathrm{~K}$ (Figure 3c), the densities of Te inclusions are reduced to the orders of $10^{3} \mathrm{~cm}^{-2}, 10^{2} \mathrm{~cm}^{-2}$ and $10^{1} \mathrm{~cm}^{-2}$. The density of Te inclusions markedly reduces when the thermal treatment temperature increases from $873 \mathrm{~K}$ to $1073 \mathrm{~K}$. Apparently, annealing under a $\mathrm{Cd}$ atmosphere is effective for eliminating inclusions. Vydyanath et al. [18] pointed out that Te inclusions reduction could be explained by the thermomigration theory. When the thermal treatment temperature is higher than $723 \mathrm{~K}$ (the melting point of $\mathrm{Te}$ ), the solid inclusion phase will transform to a liquid phase. The liquid inclusion phase migrates to the crystal surface and then will be eliminated by evaporation or reaction. Moreover, in this paper, Te inclusions cannot be eliminated completely when the temperature of annealing under $\mathrm{Cd}$ atmosphere is $1073 \mathrm{~K}$ and the time is more than $240 \mathrm{~h}$. This result is consistent with that of our previous research [17]. According to the dynamics of thermal migration [19], Te inclusions with a small size were hard to migrate because of big surface energy. The size of some Te inclusions for CMT crystals investigated in this paper is only several microns or even about $1 \mu \mathrm{m}$. In the end, although the annealing takes a long time, these inclusions are still unable to be eliminated.

For clearly observing the morphology of the inclusions further, IR microscope images of the same CMT:In slice before and after Cd atmosphere annealing ( $873 \mathrm{~K}$ for $240 \mathrm{~h}$ ) and Te atmosphere 
re-annealing ( $773 \mathrm{~K}$ for $120 \mathrm{~h}$ ) are shown in Figure 4 . In Figure $4 \mathrm{~A}-\mathrm{C}$, the density of Te inclusions gradually decreases. However, the density is still in the order of $10^{3} \mathrm{~cm}^{-2}$. In the enlarged images, the size of Te inclusions is smaller and smaller. Te inclusions can also be eliminated by annealing under Te atmosphere. A small number of Te inclusions still migrate when the annealing takes place under a Te atmosphere. The process is different from that of annealing under a $\mathrm{Cd}$ atmosphere. A Te atmosphere hinders the migration of Te inclusions, which results in few Te inclusions being eliminated. Moreover, the morphology of the inclusions is spherical after annealing. The reason is that Te droplets form during annealing and then the shape is maintained during cooling [20].

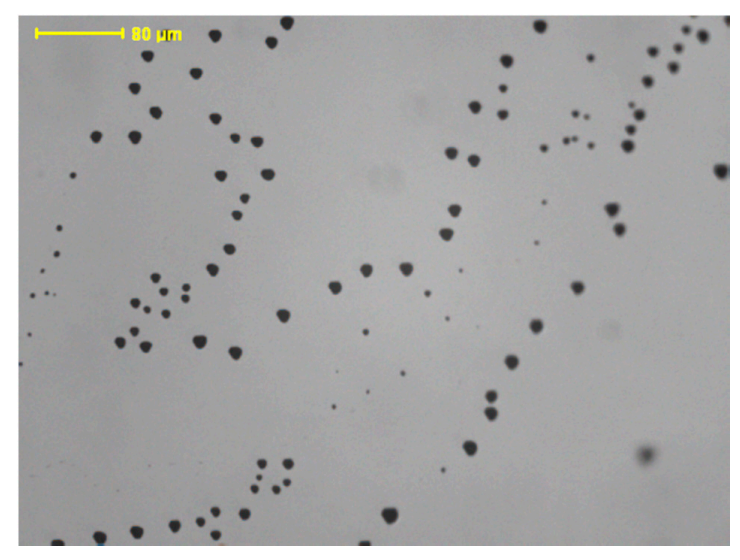

Figure 2. The typical IR image of an as-grown CMT:In crystal.

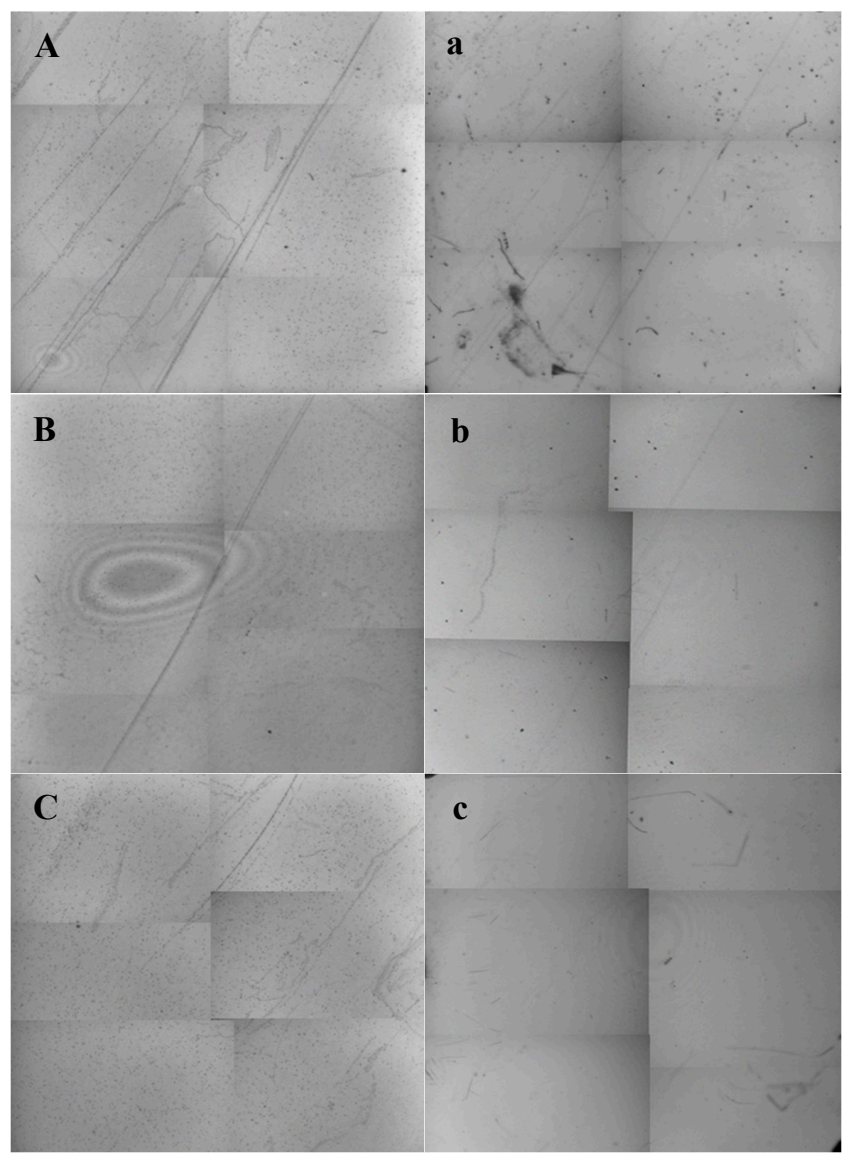

Figure 3. IR images of CMT:In crystals before and after Cd atmosphere annealing: (A-C) as-grown; (a) $873 \mathrm{~K}$ for $240 \mathrm{~h}$; (b) $973 \mathrm{~K}$ for $240 \mathrm{~h}$; (c) $1073 \mathrm{~K}$ for $240 \mathrm{~h}$. The size of the images is $9 \times 9 \mathrm{~mm}^{2}$. 

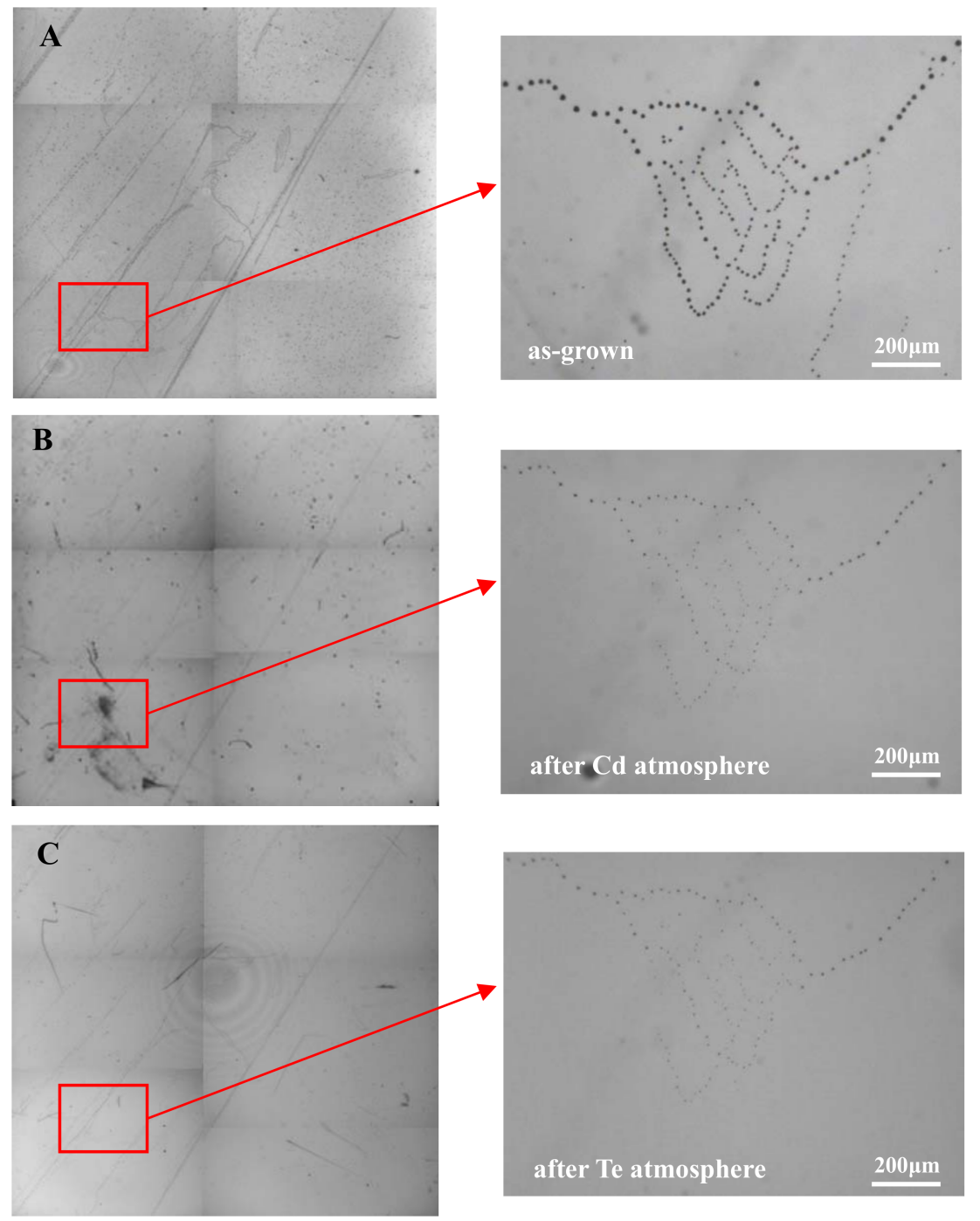

Figure 4. IR images of CMT:In crystals before and after annealing: (A) as-grown; (B) after Cd atmosphere annealing at $873 \mathrm{~K}$ for $240 \mathrm{~h}$; (C) after Te atmosphere annealing at $773 \mathrm{~K}$ for $120 \mathrm{~h}$. The size of the images is $9 \times 9 \mathrm{~mm}^{2}$.

\subsection{Effect of Multi-Step Annealing on Crystal Quality}

An X-ray rocking curve is used to test the crystal quality of CMT before and after annealing, as shown in Figure 5. The result of the full-width at-half-maximum (FWHM) for the as-grown CMT:In crystal is $68^{\prime \prime}$. Then, the value for the crystal after annealing decreases to $37^{\prime \prime}$. Moreover, the corresponding intensity of the diffraction peak improves, and the position moves from $11.5945^{\circ}$ to $11.5875^{\circ}$. Obviously, the result of the X-ray rocking curve indicates that the crystal quality is improved after annealing. Generally, the defect or macro elastic stress in crystals will destroy crystal structure perfection and cause the irregular array of atoms. In consequence, the crystal plane distorts and inclines, which results in the change of interplanar spacing. Thus, structure defects, such as inclusions and dislocations, will cause lattice distortion and the change of interplanar spacing. In this paper, many Te inclusions and dislocations exist in the as-grown crystal. These defects cause mismatch stress and destroy crystal structure. According to the Bragg's law [17], the diffraction angle of an X-ray changes and the diffraction peak deviates from the theoretical position. The FWHM is wide and the intensity is weak. For the annealed crystal with fewer defects, the FWHM becomes narrow, the intensity is enhanced, and the diffraction peak moves closer to the theoretical position. 


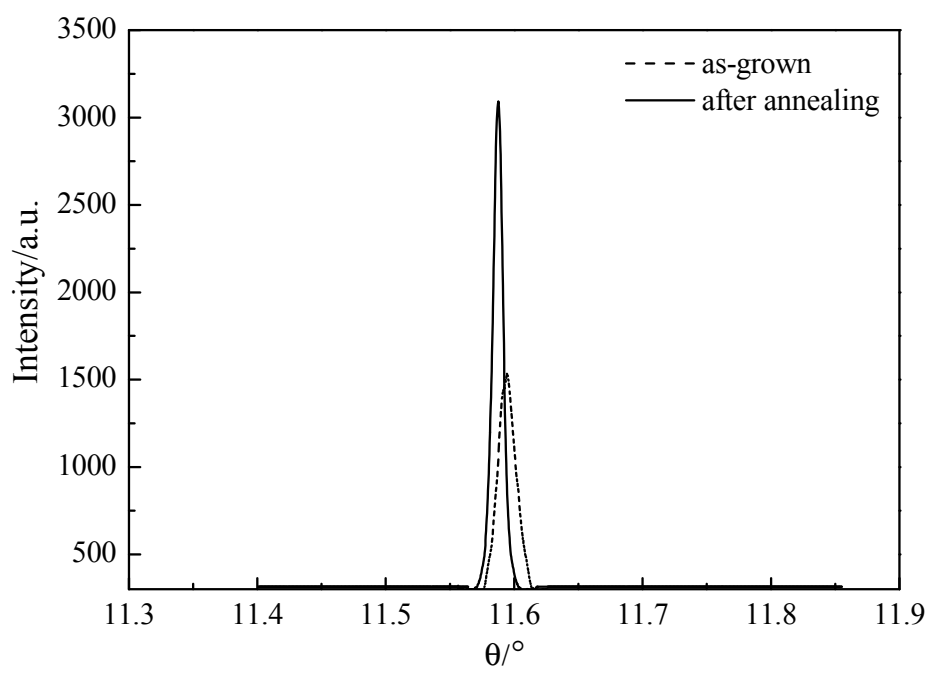

Figure 5. X-ray rocking curves of CMT:In slices, before and after annealing.

\subsection{Effect of Multi-Step Annealing on Resistivity}

For current-voltage (I-V) measurement, Au electrodes are deposited on both sides of the slices. Figure 6 shows the results of I-V measurements for CMT:In slices before and after annealing. The resistivity for as-grown CMT:In crystal is about $10^{9} \Omega \cdot \mathrm{cm}$ order of magnitude. The leakage current is within $21 \mathrm{nA}$ when the bias voltage is $100 \mathrm{~V}$. After annealing under a $\mathrm{Cd}$ atmosphere, the variation of the leakage current is noticeable (Figure $6 \mathrm{~b}$ ). A significant decrease of the resistivity is observed. When bias voltage is higher than $5 \mathrm{~V}$, the leakage current rapidly increases and reaches up to $\mu \mathrm{A}$ order of magnitudes. Hall measurement indicates that the crystal exhibits n-type conduction. Lyahovitskaya et al. [21] pointed out that the concentration of electrons increased after $\mathrm{Cd}$ atmosphere annealing and resulted in n-type conduction. Actually, $\mathrm{Cd}$ atoms diffuse into CMT:In crystal to form $\mathrm{Cd}$ interstitial atoms $\left(\mathrm{Cd}_{\mathrm{i}}\right)$ during $\mathrm{Cd}$-rich annealing. Thus, $\mathrm{Cd}$ vacancies $\left(\mathrm{V}_{\mathrm{Cd}}\right)$ are wiped out. The concentration of the n-type carrier increases and material becomes n-type conduction. In Figure $6 \mathrm{~b}$, the I-V curve is nonsymmetrical. In fact, Cd will diffuse to both sides of CMT crystal, but more $\mathrm{Cd}$ atoms will diffuse to the side that is close to the $\mathrm{Cd}$ source, which will result in asymmetry of the I-V curve. For a long annealing time, $\mathrm{Cd}$ atoms will diffuse to the whole crystal and the I-V curve will be symmetrical. When bias voltage is higher than $50 \mathrm{~V}$, the leakage current is large and reaches the limit of the equipment. After re-annealing under Te atmosphere, the resistivity is enhanced markedly (Figure 6c). This result achieves the purpose of keeping high resistivity. It can be seen that the leakage current is only $3 \mathrm{nA}$. The high-resistivity material is formed. Te saturated vapor pressure restrains the evaporation of the Te element in CMT crystal. $\mathrm{Cd}_{\mathrm{i}}$ atoms decrease and $\mathrm{V}_{\mathrm{Cd}}$-acceptor concentration is increased by the evaporation of the $\mathrm{Cd}$ element. Hall measurement indicates that the carrier concentration of the annealed CMT:In slice is $10^{4} \mathrm{~cm}^{-3}$ order of magnitudes and the slice has weak p-type conduction. Therefore, the reduction of the carrier concentration results in high resistivity. The resistivities of $873 \mathrm{~K}, 973 \mathrm{~K}$, and $1073 \mathrm{~K}$ in annealed CMT:In crystals are calculated to be $1.26 \times 10^{10} \Omega \cdot \mathrm{cm}, 2.24 \times 10^{10} \Omega \cdot \mathrm{cm}$ and $2.75 \times 10^{10} \Omega \cdot \mathrm{cm}$, respectively, which are higher than that of as-grown crystals. 

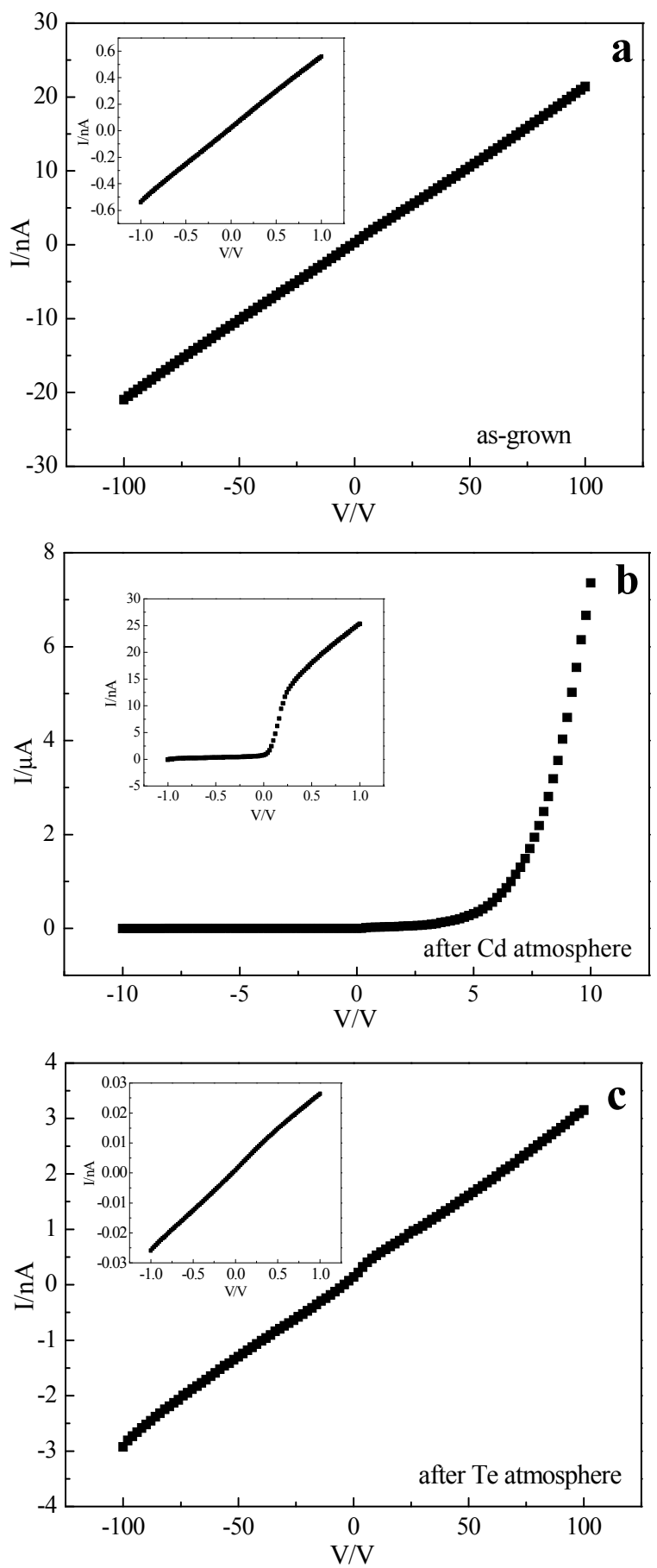

Figure 6. Current-voltage (I-V) curves of CMT:In crystals before and after annealing: (a) as-grown; (b) after $\mathrm{Cd}$ atmosphere; (c) after Te atmosphere. The inserts are the I-V curves $(-1 \mathrm{~V} 1 \mathrm{~V})$ of the crystals.

\subsection{Effect of Multi-Step Annealing on IR Transmittance Spectra}

IR transmittance spectrum is also used to evaluate the crystal quality of CMT crystals. Figure 7 shows the test result. In Figure 7a, the transmittance curves are high straight lines for both as-grown and annealed CMT:In slices in the wave number region from $500 \mathrm{~cm}^{-1}$ to $4000 \mathrm{~cm}^{-1}$. The average transmittance for as-grown crystal is $53 \%$. However, the transmittance curves for $\mathrm{Cd}$ atmosphere annealed crystals show ascending lines. The average transmittances are 33\% (873 K), 29\% (973 K) and $27 \%(1073 \mathrm{~K})$, respectively. Hall measurement indicates that the carrier concentration for as-grown crystals is $3.16 \times 10^{6} \mathrm{~cm}^{-3}$. However, the concentration of carriers for the crystal after $\mathrm{Cd}$ atmosphere 
annealing at $1073 \mathrm{~K}$ is $6.44 \times 10^{14} \mathrm{~cm}^{-3}$. The carrier concentration increases tremendously. The higher the annealing temperature, the more the concentration of carriers there is. Here, the free-carrier absorption dominates the IR absorption and influences IR transmittance [22,23]. This result is consistent with that of electrical measurement after $\mathrm{Cd}$ atmosphere annealing.
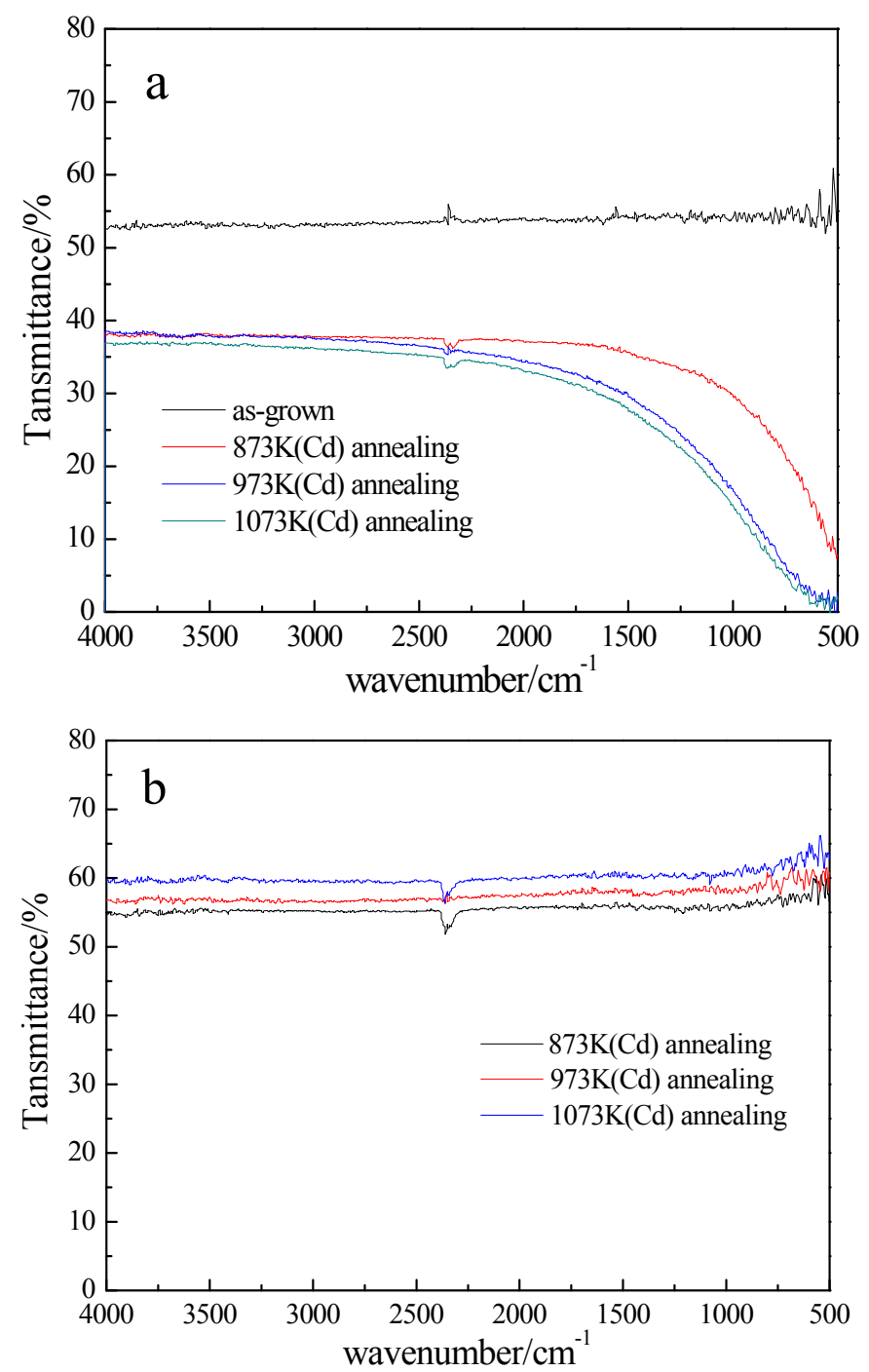

Figure 7. IR transmittance curves of CMT:In crystals before and after annealing: (a) Cd atmosphere;

(b) Te atmosphere.

In Figure $7 \mathrm{~b}$, the transmittance curves of the CMT:In slices show the feature of high straight lines again after re-annealing under Te atmosphere. Hall measurement indicates that the carrier concentration is $5.68 \times 10^{4} \mathrm{~cm}^{-3}$. It is clear that the carrier concentration is greatly decreased. The result is still consistent with that of electrical measurement after Te atmosphere annealing. The free-carrier absorption does not dominate the IR absorption because the carrier concentrations are low for all slices.

\subsection{Effect of Multi-Step Annealing on PL Spectra}

Photoluminescence (PL) spectroscopy is used to characterize point defects in CMT:In crystals. It is helpful to understand the annealing influence. The low-temperature PL spectra at $10 \mathrm{~K}$ for as-grown and annealed CMT:In slices is shown in Figure 8. The spectra can be divided into three regions according to Du et al. [22]. They are near-band-edge region (1.68-1.76 eV), donor-acceptor pair $(\mathrm{D}, \mathrm{A})$ region (1.59-1.68 eV), and A-center region (1.42-1.59 eV). 


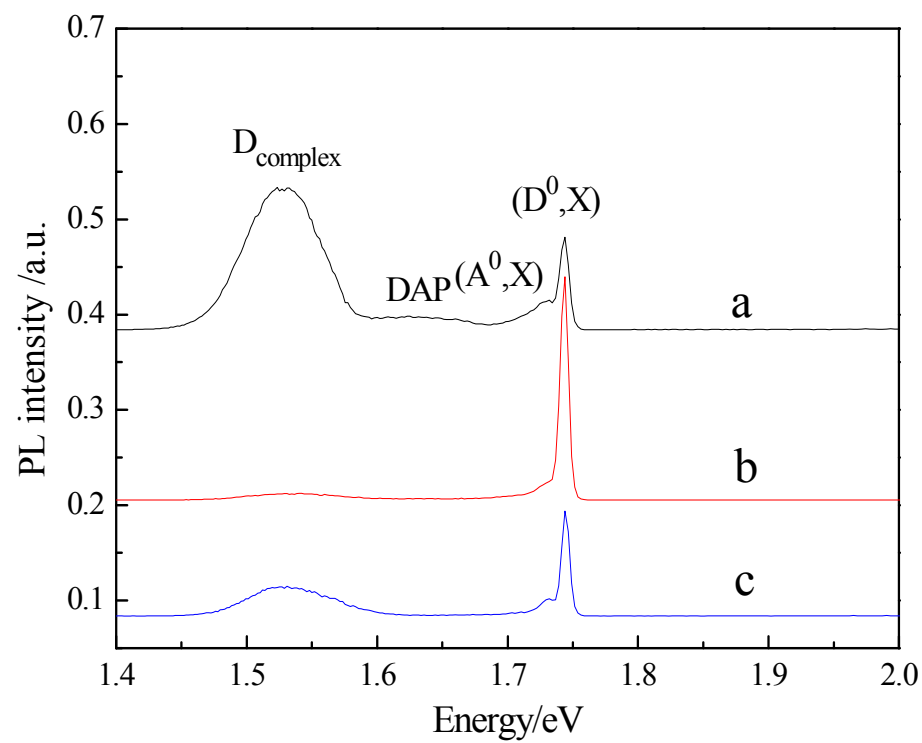

Figure 8. Photoluminescence (PL) spectra of CMT:In crystals before and after annealing: (a) as-grown (b) Cd atmosphere; (c) Te atmosphere.

Comparing curve a with curve $\mathrm{b}$ in Figure 8, some interesting changes are found. In the near-band-edge region, the $\left(\mathrm{A}^{0}, \mathrm{X}\right)$ peak almost disappears after $\mathrm{Cd}$ atmosphere annealing. The intensity of the $\left(\mathrm{D}^{0}, \mathrm{X}\right)$ peak significantly increases and the FWHM reduces. Generally, the crystal quality depends on the intensity of the neutral donor bound exciton peak $\left(\mathrm{D}^{0}, \mathrm{X}\right) . \mathrm{Cd}$ atmosphere annealing effectively eliminates Te inclusions and improves the crystal quality. In the donor-acceptor pair (DAP) region, a DAP peak can be observed in as-grown crystal, while it disappears after annealing. The phenomenon can be explained by the removal of the impurities [17,22]. Moreover, the DAP peak is relative to the complexes formed by indium and Cd vacancy [24]. Cd atoms diffuse into CMT:In crystals and compensate $\mathrm{Cd}$ vacancies in the complexes. This can also decrease the intensity of the DAP peak. Finally, in the A-center region, the intensity of the $\mathrm{D}_{\text {complex }}$ peak remarkably decreases after annealing. $A D_{\text {complex }}$ peak is generally ascribed to $C d$ vacancy-shallow donor complex [22]. In indium-doped CdTe-based crystals, ionized donor $\left[\mathrm{In}_{\mathrm{Cd}}\right]^{+}$and $\mathrm{Cd}$ vacancy related complex is responsible for the $\mathrm{D}_{\text {complex }}$ peak [25]. Two factors, the compensation of $\mathrm{Cd}$ vacancies and the elimination of Te inclusions, result in the reduction of the intensity of the $\mathrm{D}_{\text {complex }}$ peak. In addition, a $\mathrm{I}\left(\mathrm{D}^{0}, \mathrm{X}\right) / \mathrm{I}\left(\mathrm{D}_{\text {complex }}\right)$ ratio is also adopted to evaluate the crystal quality. The larger the ratio, the better the crystal quality is. The ratios for as-grown and annealed crystals are 1.27 and 4.70, respectively. It also shows the improvement of the crystal quality. After re-annealing under Te atmosphere, comparing curve $b$ with curve $c$ in Figure 8, there are two obvious changes. First, the intensity of the $\left(D^{0}, X\right)$ peak decreases, and second the $\mathrm{D}_{\text {complex }}$ peak increases. It can be explained by the increase of $\mathrm{Cd}$ vacancy density in the process of Te atmosphere annealing [22]. After Te atmosphere annealing, the $\mathrm{I}\left(\mathrm{D}^{0}, \mathrm{X}\right) / \mathrm{I}\left(\mathrm{D}_{\text {complex }}\right)$ ratio varies from 4.70 to 1.69 . However, on the whole, the crystal quality is improved after annealing. Figure 9 shows the PL spectra of CMT:In crystals after multi-step annealing. It can be seen that the intensity of the $\left(\mathrm{D}^{0}, \mathrm{X}\right)$ peak increases and the intensity of the $\mathrm{D}_{\text {complex }}$ peak decreases as the annealing temperature increases. The corresponding value of the $\mathrm{I}\left(\mathrm{D}^{0}, \mathrm{X}\right) / \mathrm{I}\left(\mathrm{D}_{\text {complex }}\right)$ ratios is also enlarged. Thus, the crystal quality is gradually improved with the increasing annealing temperature. 


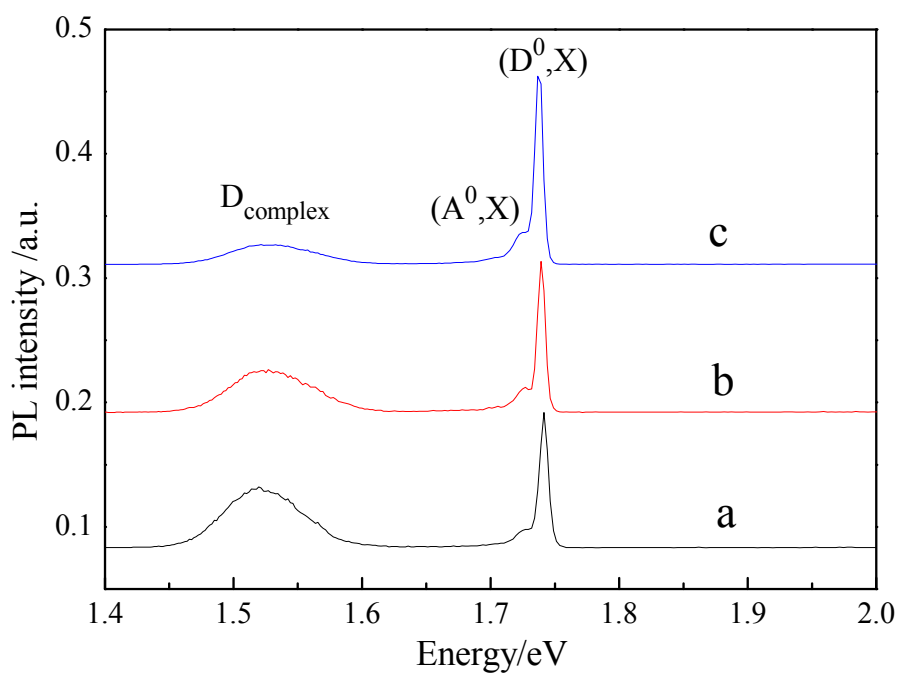

Figure 9. PL spectra of CMT:In crystals after multi-step annealing: (a) Cd atmosphere annealing at $873 \mathrm{~K}$ and Te annealing re-annealing at $773 \mathrm{~K}$; (b) Cd atmosphere annealing at $973 \mathrm{~K}$ and Te annealing re-annealing at $773 \mathrm{~K}$; (c) Cd atmosphere annealing at $1073 \mathrm{~K}$ and Te annealing re-annealing at $773 \mathrm{~K}$.

\subsection{Effect of Multi-Step Annealing on the Performance of Detectors}

The as-grown and annealed CMT:In slices are fabricated to room temperature detectors after the preparation of planar-electrodes on both sides. Figure 10 shows ${ }^{241} \mathrm{Am} \gamma$-ray spectra of the detectors. The applied voltage is $60 \mathrm{~V}$, the shaping time is $1 \mu \mathrm{s}$. The energy resolutions of the detectors fabricated by three as-grown CMT:In slices are 35.38\% (Figure 10a), 36.27\% (Figure 10b) and 36.94\% (Figure 10c), respectively. Meanwhile, the corresponding energy resolutions of the detectors fabricated by annealed CMT:In slices are 14.79\% (Figure 10a), 11.18\% (Figure 10b) and $9.43 \%$ (Figure 10c), respectively. Apparently, the energy resolution is drastically improved.

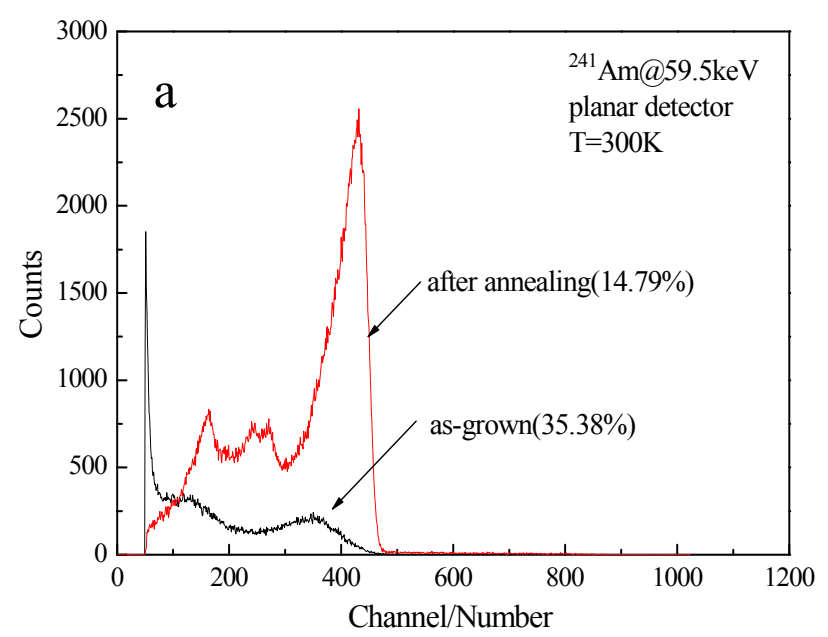

Figure 10. Cont. 

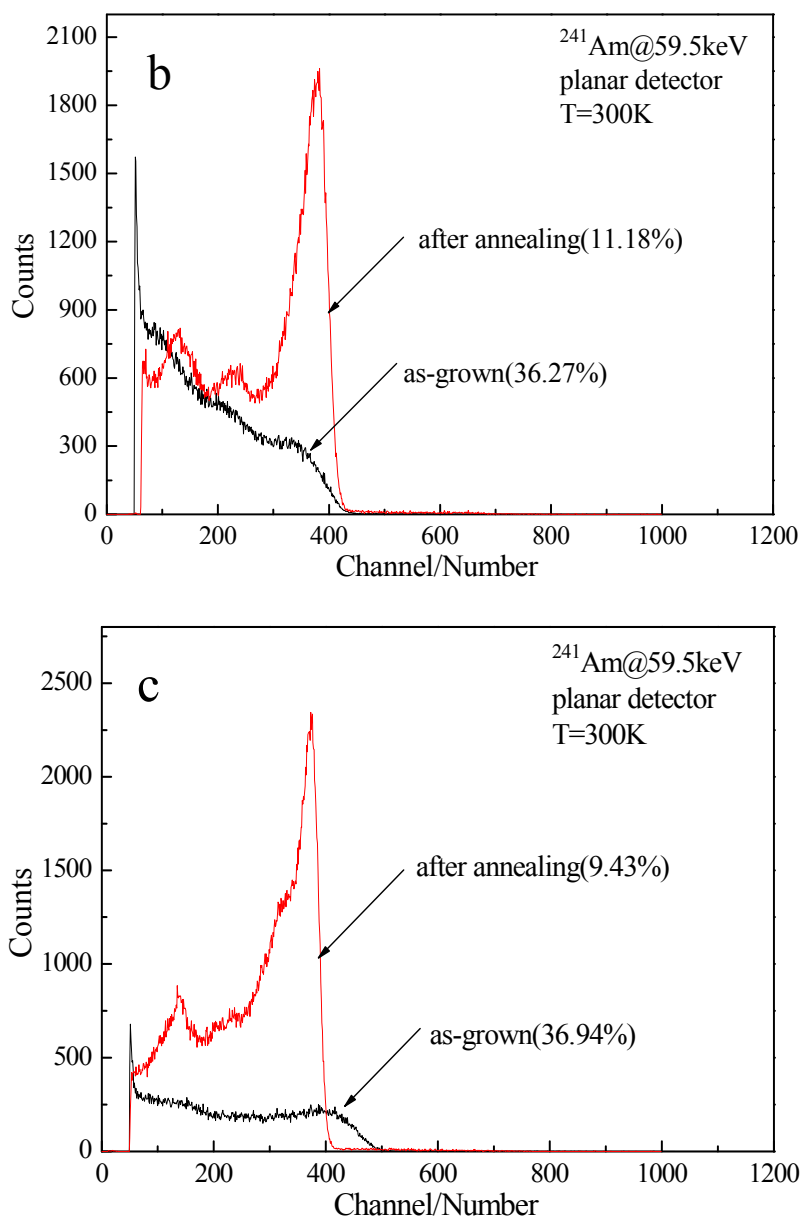

Figure 10. ${ }^{241} \mathrm{Am} \gamma$-ray spectra of CMT detectors fabricated by as-grown and annealed CMT:In slices: (a) Cd atmosphere at $873 \mathrm{~K}$ for $240 \mathrm{~h}$ and Te atmosphere at $773 \mathrm{~K}$ for $120 \mathrm{~h}$; (b) Cd atmosphere at $973 \mathrm{~K}$ for $240 \mathrm{~h}$ and Te atmosphere at $773 \mathrm{~K}$ for $120 \mathrm{~h}$; (c) $\mathrm{Cd}$ atmosphere at $1073 \mathrm{~K}$ for $240 \mathrm{~h}$ and Te atmosphere at $773 \mathrm{~K}$ for $120 \mathrm{~h}$.

The Hecht Equation (1) [26] is used to calculate the mobility-lifetime products of single carriers (electron or hole), as follows:

$$
Q=Q_{0} \times \frac{\mu \tau E}{d}\left[1-\exp \left(\frac{-d}{\mu \tau E}\right)\right],
$$

where $Q$ and $Q_{0}$ are the charge collection and the maximum collectible charge, respectively; $\mu \tau$ is the mobility-lifetime product; $E$ is the applied electric field, and $d$ is the thickness of the sample. As the voltage changes, the energy resolution and the position of the energy peak are also changed. Based on this, the collection efficiency of the detectors is determined. Then the $\mu \tau$ value for single carriers can also be calculated. The defects which are trapping centers will influence the mobility-lifetime product. For as-grown crystal, there are lots of Te inclusions and dislocations. Low resistivity will result in high leakage current, which causes high electrical noise. Therefore, the detectors fabricated by as-grown crystals have low collection efficiency and poor energy resolution. Thus, the $\mu \tau$ value of an electron for as-grown crystal cannot be calculated because there is not enough data of the voltage vs the peak position. After annealing, the defects are observably decreased and the resistivity is enhanced. As a consequence, the energy resolution improves. The collection efficiency of the detector (fabricated by the slice after $\mathrm{Cd}$ atmosphere annealing at $1073 \mathrm{~K}$ for $240 \mathrm{~h}$ and Te atmosphere re-annealed at $773 \mathrm{~K}$ for $120 \mathrm{~h}$ ) at different voltages is shown in Figure 11. The $\mu \tau$ values of the detectors fabricated by annealed CMT:In slices are $2.50 \times 10^{-4} \mathrm{~cm}^{2} / \mathrm{V}\left(873 \mathrm{~K}\right.$, Figure 10a), $7.58 \times 10^{-4} \mathrm{~cm}^{2} / \mathrm{V}(973 \mathrm{~K}$, Figure 10b) and $1.25 \times 10^{-3} \mathrm{~cm}^{2} / \mathrm{V}(1073 \mathrm{~K}$, Figure 10c), respectively. The detector with the best performance can 
achieve the requirement of high-performance detection. It is found that the mobility-lifetime product for as-grown CMT is about $10^{-3} \mathrm{~cm}^{2} / \mathrm{V}$ orders of magnitude [27], significantly less than that (about $10^{-2} \mathrm{~cm}^{2} / \mathrm{V}$ order of magnitudes) for as-grown CdZnTe [28]. There are mainly two reasons for this problem. First, the purity of Mn $(5 \mathrm{~N})$ is markedly lower than that of $\mathrm{Zn}(7 \mathrm{~N})$. Second, CMT crystal with sphalerite structure has very low layer fault energy, which will result in the formation of more twins. Thus, more impurities and defects in as-grown CMT crystals reduce the mobility-lifetime product. Bulk CdZnTe with a high quality can be grown by high quality seeds. However, CMT seeds with a high quality are difficult to obtain. Moreover, compared to CdZnTe, the detector performance of the crystals obtained by post-growth annealing is also not good because CMT has more defects. In our previous work [29], Te inclusions could be eliminated completely for CdZnTe, but this defect could not be eliminated completely for CMT [30]. The best fitting for the $\mu \tau$ value is shown in Figure 11 . However, there are still errors between the experimental and the theoretical values. It is known that the mobility-lifetime products are mainly influenced by defects. Defects, such as Te inclusions, will cause an inhomogeneous distribution of electric field. The migration of carriers will be influenced, which results in the deviation between the experimental and the theoretical values. Actually, the improvement of the detector performance is due to the enhancement of the crystal quality. The decrease of trapping centers improves the transmission efficiency of carriers, and high resistivity guarantees good energy resolution. The optimal annealing condition for CMT:In crystal is Cd atmosphere annealing at $1073 \mathrm{~K}$ for $240 \mathrm{~h}$ and re-annealing under Te atmosphere at $773 \mathrm{~K}$ for $120 \mathrm{~h}$.

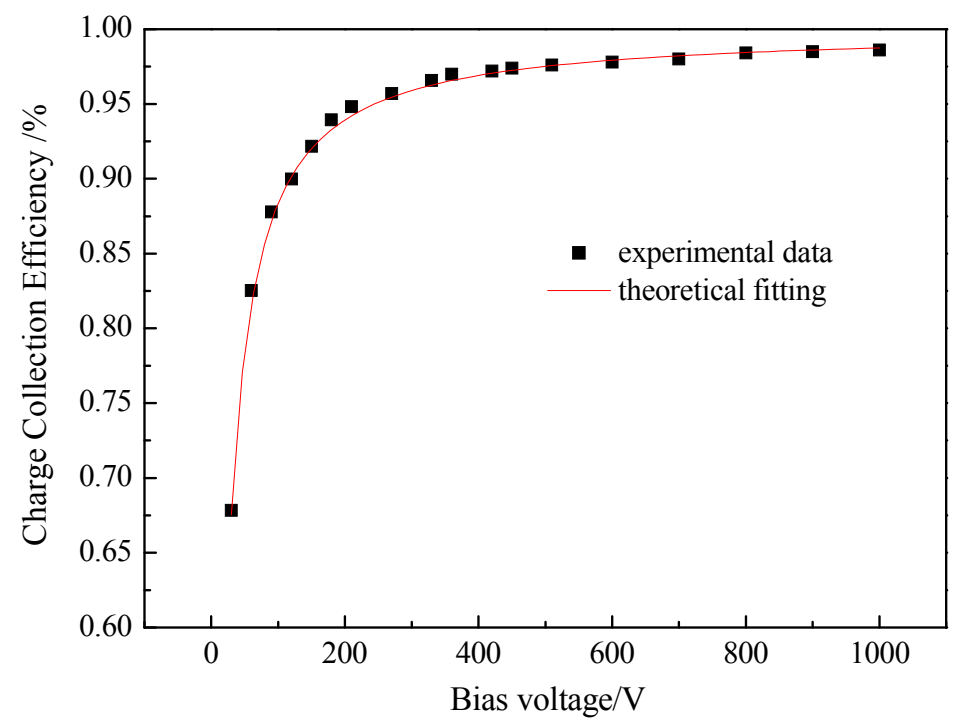

Figure 11. Collection efficiency of CMT:In detectors after Cd atmosphere annealing at $1073 \mathrm{~K}$ for $240 \mathrm{~h}$ and Te atmosphere re-annealing at $773 \mathrm{~K}$ for $120 \mathrm{~h}$.

\section{Conclusions}

The main effects of a multi-step annealing method on secondary phase particles, related defects, and resistivity of high-resistivity CMT:In crystals were investigated. The method included a Cd atmosphere annealing step and a Te atmosphere annealing step. After annealing under a Cd atmosphere, most Te inclusions were eliminated. Re-annealing under a Te atmosphere could also reduce this defect. The decrease of the FWHM of an X-ray rocking curve and the increase of IR transmittance indicated the improvement of the crystal quality. PL measurements also showed that the crystal quality was improved by the increase of the intensity of the $\left(\mathrm{D}^{0}, \mathrm{X}\right)$ peak, the disappearance of the DAP peak and the decrease of the $\mathrm{D}_{\text {complex }}$ peak. After annealing, the detector performance was obviously improved. The higher the annealing temperature, the better the performance was. The best detector performance was a $9.43 \%$ energy resolution and a $1.25 \times 10^{-3} \mathrm{~cm}^{2} / \mathrm{V} \mu \tau$ value. The optimal 
condition of thermal treatment in this paper was Cd atmosphere annealing at $1073 \mathrm{~K}$ for $240 \mathrm{~h}$ and re-annealing under Te atmosphere at $773 \mathrm{~K}$ for $120 \mathrm{~h}$.

Author Contributions: Analysis and writing, P.Y.; experiments and characterization, Y.C., W.L. (Wei Li), W.L. (Wenfei Liu), B.L., J.Y. and K.N.; project administration, W.J.; conceptualization and methodology, L.L., J.Z., Z.L., M.B., G.S., and H.L.

Funding: This research was funded by the National Natural Science Foundations of China (Grant Nos. 51602026, 51608041, 21607013 and 11604022), the Natural Science Basic Research Plan in Shaanxi Province of China (No. 2018JM5150), the China Postdoctoral Science Foundation (Grant No. 2014M550509), the Special Fund for Basic Scientific Research of Central Colleges of Chang'an University (Nos. 300102318106, 300102318202, 310831171014), and Undergraduate Training Programs for Innovation and Entrepreneurship of Chang'an University (No. 201710710274).

Conflicts of Interest: The authors declare no conflicts of interest.

\section{References}

1. Parkin, J.; Sellin, P.J.; Davies, A.W.; Lohstroh, A.; Özsan, M.E.; Seller, P. $\alpha$ Particle response of undoped CdMnTe. Nucl. Instrum. Meth. A 2007, 573, 220-223. [CrossRef]

2. Hossain, A.; Cui, Y.; Bolltnikov, A.E.; Camarda, G.S.; Yang, G.; Kochanowska, D.; Witkowska-baran, M.; Mycielski, A.; James, R.B. Vanadium-doped cadmium manganese telluride $\left(\mathrm{Cd}_{1-\mathrm{x}} \mathrm{Mn}_{\mathrm{x}} \mathrm{Te}\right)$ crystals as $\mathrm{x}$ - and gamma-ray detectors. J. Electron. Mater. 2009, 38, 1593-1599. [CrossRef]

3. Roy, U.N.; Camarda, G.S.; Cui, Y.; Gu, G.; Gul, R.; Hossain, A.; Yang, G.; Egarievwe, S.U.; James, R.B. Growth and characterization of CdMnTe by the vertical Bridgman technique. J. Cryst. Growth 2016, 437, 53-58. [CrossRef]

4. Babalola, O.S.; Bolotnikov, A.E.; Groza, M.; Hossain, A.; Egarievwe, S.; James, R.B.; Burger, A. Study of Te inclusions in CdMnTe crystals for nuclear detector applications. J. Cryst. Growth 2009, 311, 3702-3707. [CrossRef]

5. Du, Y.Y.; Jie, W.Q.; Zheng, X.; Wang, T.; Bai, X.X.; Yu, H. Growth interface of In-doped CdMnTe from Te solution with vertical Bridgman method under ACRT technique. Trans. Nonferrous Met. Soc. China 2012, 22, s143-s147. [CrossRef]

6. Hossain, A.; Gu, G.D.; Bolltnikov, A.E.; Camarda, G.S.; Cui, Y.; Yang, G.; Roy, U.N.; Yang, G.; Liu, T.; Zhong, R.; et al. Material and detector properties of cadmium manganese telluride $\left(\mathrm{Cd}_{1-\mathrm{x}} \mathrm{Mn} \mathrm{n}_{\mathrm{x}} \mathrm{Te}\right)$ crystals grown by the modified floating-zone method. Nucl. Instrum. Meth. A 2015, 784, 33-36. [CrossRef]

7. Kochanowska, D.; Mycielski, A.; Witkowska-Baran, M.; Szadkowski, A.; Witkowska, B.; Kaliszek, W.; Cui, Y.; James, R.B. Influence of annealing on tellurium precipitates in (Cd,Mn)Te:V crystals. IEEE Nucl. Sci. Symp. Conf. Rec. 2009, NSS'08, 202-206.

8. Zhang, J.J.; Wang, L.J.; Min, J.H.; Qin, K.F.; Huang, J.; Tang, K.; Liang, X.Y.; Shen, P. Annealing of indium-doped CdMnTe single crystals under Cd vapors. J. Cryst. Growth 2012, 358, 12-15. [CrossRef]

9. Zhang, J.J.; Wang, L.J.; Min, J.H.; Qin, K.F.; Shi, Z.B.; Liang, X.Y. Te vapor annealing of indium-doped CdMnTe crystals. J. Semicond. 2015, 34, 033002. [CrossRef]

10. Adams, A.L.; Agbalagba, E.O.; Jow, J.O.; Mwathi, J.G.; Egarievwe, A.A.; Chan, W.; Dowdell, M.C.; Roy, U.N.; Egarievwe, S.U. Thermal annealing of CdMnTe material being developed for nuclear radiation detection applications. IOSR J. Mech. Civ. Eng. 2016, 14, 1-5. [CrossRef]

11. Bugár, M.; Belas, E.; Procházka, J.; Hlídek, P.; Grill, R.; Franc, J.; Höschl, P. IR transmittance of CdTe after high-temperature annealing. Nucl. Instrum. Meth. A 2011, 633, S83-S85. [CrossRef]

12. Kim, K.; Bolotnikov, A.E.; Camarda, G.S.; Hossain, A.; James, R.B. Overcoming Zn segregation in CdZnTe with the temperature gradient annealing. J. Cryst. Growth 2016, 442, 98-101. [CrossRef]

13. Egarievwe, S.U.; Yang, G.; Egarievwe, A.A.; Okwechime, I.O.; Gray, J.; Hales, Z.M.; Hossain, A.; Camarda, G.S.; Bolotnikov, A.E.; James, R.B. Post-growth annealing of Bridgman-grown CdZnTe and CdMnTe crystals for room-temperature nuclear radiation detectors. Nucl. Instrum. Meth. A 2015, 784, 51-55. [CrossRef]

14. Chu, M.; Terterian, S.; Ting, D.; Wang, C.C.; Dinan, J.H.; James, R.B.; Burger, A. Effects of excess tellurium on the properties of CdZnTe radiation detectors. J. Electron. Mater. 2003, 32, 779-782. [CrossRef] 
15. Yang, G.; Bolotnikov, A.E.; Fochuk, P.M.; Kopach, O.; Franc, J.; Belas, E.; Kim, K.H.; Camarda, G.S.; Hossain, A.; Cui, Y.; et al. Post-growth thermal annealing study of CdZnTe for developing room-temperature X-ray and gamma-ray detectors. J. Cryst. Growth 2013, 379, 16-20. [CrossRef]

16. Li, G.Q.; Zhang, X.L.; Jie, W.Q.; Hui, C. Thermal treatment of detector-grade CdZnTe. J. Cryst. Growth 2006, 295, 31-35. [CrossRef]

17. Yu, P.F.; Xu, Y.D.; Luan, L.J.; Du, Y.Y.; Zheng, J.H.; Li, H.; Jie, W.Q. Quality improvement of CdMnTe:In single crystals by an effective post- growth annealing. J. Cryst. Growth 2016, 451, 194-199. [CrossRef]

18. Vydyanath, H.R.; Ellsworth, J.A.; Parkinson, J.B.; Kennedy, J.J.; Dean, B.; Johnson, C.J.; Neugebauer, G.T.; Sepich, J.; Liao, P. Thermomigration of Te precipitates and improvement of $(\mathrm{Cd}, \mathrm{Zn}) \mathrm{Te}$ substrate characteristics for the fabrication of LWIR (Hg, Cd)Te photodiodes. J. Electron. Mater. 1993, 22, 1073-1080. [CrossRef]

19. Anthony, T.R.; Cline, H.E. Thermal migration of liquid droplets through solids. J. Appl. Phys. 1971, 42, 3380-3387. [CrossRef]

20. Yu, P.F.; Jie, W.Q. Observation, morphology evolution and elimination of Te inclusions in CdZnTe:In single crystals. J. Cryst. Growth 2013, 381, 57-60. [CrossRef]

21. Lyahovitskaya, V.; Chernyak, L.; Greenberg, J.; Kaplan, L.; Cahen, D. n- And p-type post-growth self-doping of CdTe single crystals. J. Cryst. Growth 2000, 214, 1155-1157. [CrossRef]

22. Du, Y.Y.; Jie, W.Q.; Wang, T.; Zheng, X.; Xu, Y.D.; Luan, L.J. Solution growth of In-doped CdMnTe crystals by the vertical Bridgman method with the ACRT technique. J. Cryst. Growth 2012, 355, 33-37. [CrossRef]

23. Yu, P.F.; Luan, L.J.; Du, Y.Y.; Zheng, J.H.; Jie, W.Q. Investigation of Te atmosphere annealing on the properties of detector-grade CdMnTe:In single crystals. J. Cryst. Growth 2015, 430, 103-107. [CrossRef]

24. Zhang, J.J.; Wang, L.J.; Min, J.H.; Huang, J.; Liang, X.Y.; Tang, K.; Shen, P.; Shen, M.; Liang, W.; Huang, N.S.; Xia, Y.B. Characterization of CdMnTe crystal grown with vertical Bridgman method under Te-rich conditions. Phys. Status Solidi C 2014, 11, 1174-1177. [CrossRef]

25. Li, Q.; Jie, W.Q.; Fu, L.; Wang, T.; Yang, G.; Bai, X.X.; Zha, G.Q. Optical and electrical properties of indium-doped $\mathrm{Cd}_{0.9} \mathrm{Zn}_{0.1}$ Te crystal. J. Cryst. Growth 2006, 295, 124-128. [CrossRef]

26. Jones, K.A.; Ciampi, G.; Skrip, C.E.; Lynn, K.G. Vertical Bridgman growth of $\mathrm{Cd}_{1-\mathrm{x}} \mathrm{Zn}_{\mathrm{x}} \mathrm{Te}$ for room temperature radiation detectors. Proc. SPIE 2007, 6706, 670607.

27. Kim, K.; Jeng, G.; Kim, P.; Choi, J.; Bolotnikov, A.E.; Camarda, G.S.; James, R.B. Influence of the cooling scheme on the performance and presence of carrier traps for CdMnTe detectors. J. Appl. Phys. 2013, 114, 063706. [CrossRef]

28. Chen, H.; Awadalla, S.A.; Iniewski, K.; Lu, P.H.; Harris, F.; Mackenzie, J.; Hasanen, T.; Chen, W.; Redden, R.; Bindley, G.; et al. Characterization of large cadmium zinc telluride crystals grown by traveling heater method. J. Appl. Phys. 2008, 103, 014903. [CrossRef]

29. Yu, P.F.; Jie, W.Q. Effects of post-growth annealing on the performance of CdZnTe: In radiation detectors with different thickness. Nucl. Instrum. Meth. A 2014, 737, 29-32. [CrossRef]

30. Yu, P.F.; Xu, Y.D.; Chen, Y.R.; Song, J.; Zhu, Y.; Zhang, M.J.; Zhang, B.G.; Wang, Y.; Li, W.; Luan, L.J.; et al. Investigation of effective annealing on CdMnTe:In crystals with different thickness for gamma-ray detectors. J. Cryst. Growth 2018, 483, 94-101. [CrossRef]

(C) 2018 by the authors. Licensee MDPI, Basel, Switzerland. This article is an open access article distributed under the terms and conditions of the Creative Commons Attribution (CC BY) license (http:/ / creativecommons.org/licenses/by/4.0/). 